\title{
Estudio de la Viscosidad en un Crudo Aditivado con Dispersantes y Disolventes Asfalténicos
}

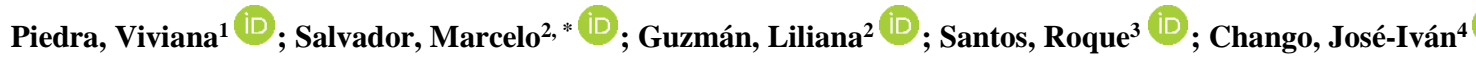 \\ ${ }^{1}$ Facultad de Ingeniería Química y Agroindustria, Escuela Politécnica Nacional, Quito, Ecuador \\ ${ }^{2}$ Facultad de Ingeniería Química y Agroindustria, Departamento de Ingeniería Química, Escuela Politécnica Nacional, Quito, \\ Ecuador \\ ${ }^{3}$ Departamento de Ciencias Nucleares, Escuela Politécnica Nacional, Quito, Ecuador \\ ${ }^{4}$ Centro de Investigaciones Aplicadas a Polímeros (CIAP), Escuela Politécnica Nacional, Quito, Ecuador
}

\begin{abstract}
Resumen: En Ecuador, el aumento de la cantidad de crudo pesado extraído genera preocupación en la industria. Debido a su naturaleza viscosa, presenta dificultades en el transporte y en la refinación. El objetivo de este proyecto es el estudio de la reducción de viscosidad de un crudo pesado, extraído del Oriente Ecuatoriano mediante el uso de compuestos dispersantes y disolventes de asfaltenos, para ser transportado dentro de un oleoducto. Para ello, se selecciona y clasifica a los reductores de viscosidad que tienen un efecto disolvente, dispersante o inhibidor de asfaltenos, por medio de sus espectros FT-IR resultantes. Se analiza el cambio de propiedades físicas y químicas del crudo con los reductores seleccionados por medio de cuatro pruebas: a) prueba para determinar la viscosidad; b) análisis S.A.R.A.; c) prueba de oliensis; d) prueba para determinar la gravedad API. Finalmente, se realiza la evaluación de la facilidad del transporte de crudo en un banco de pruebas de tuberías que presenta comportamientos similares al transporte del crudo por un oleoducto. Por medio de la clasificación y selección de los reductores, se escoge a los tres aditivos usados para las pruebas: M01-X, M02-A y M03-S. Se clasifica, a los dos primeros como disolventes de asfaltenos y el último como dispersante de asfaltenos. En la evaluación del cambio de propiedades del crudo el mejor resultado se obtiene con el aditivo "M01-X" con un porcentaje de reducción de viscosidad de 65,0 \% y aumento del caudal del crudo de 1,24 a 2,01 (mL/s). Como alternativa y mejor resultado a este reductor se usó una mezcla formada por dos aditivos (M02-A + M03-S) con un porcentaje de reducción de viscosidad de 69,0 \% y un aumento de caudal a $2,72(\mathrm{~mL} / \mathrm{s})$.
\end{abstract}

Palabras clave: aditivo, asfaltenos, oleoducto, viscosidad.

\section{Viscosity Study in a Crude Oil Additive with Dispersants and Asphaltene Solvent}

\begin{abstract}
In Ecuador, increasing quantities in the extraction of heavy crude oil has brought concern in the industry. Due to its viscous nature. This oil presents difficulties in transportation and refining. The objective of this project is the reduction of viscosity of this type of oil, that is extracted from the Ecuadorian Amazon and it is to be transported within a pipeline. The reduction in viscosity will be obtained from dispersing compounds and solvents in the oil example. To do this, viscosity reducers that had a solvent, dispersant or inhibitor effect on asphaltenes are selected and classified using their resulting FT-IR spectra. The change in physical and chemical properties of the crude oil is analyzed with the selected reducers. Four tests are carried out: a) test to determine the viscosity; b) analysis S.A.R.A ; c) oliensis test; d) test to determine API gravity. Finally, evaluation of crude oil transportation easiness is carried out in a pipeline bank that imitates crude oil transport through a pipeline. Through the classification and selection of the reducers. Three additives used for the tests are chosen: M01-X, M02-A and M03-S. The first two are classified as asphaltene solvents and the last one as an asphaltene dispersing. Regarding the evaluation of the change in crude oil properties. The best result is obtained with the additive "M01-X" with a viscosity reduction percentage of $65.0 \%$, increase in the flow rate of the crude oil from 1.24 to $2.01(\mathrm{~mL} / \mathrm{s})$. As an alternative to this reducer, a better result is obtained with the mixture formed by two additives (M02-A + M03-S) with a percentage reduction in viscosity of $69.0 \%$ and an increase in flow rate to $2.72(\mathrm{~mL} / \mathrm{s})$.
\end{abstract}

Keywords: additive, asphaltenes, pipeline, viscosity.

\section{INTRODUCCIÓN}

Las actividades relacionadas con la explotación del crudo pesado tienden a generar problemas económicos y ambientales. Respecto a los problemas económicos, se puede nombrar al aumento de importaciones de crudo liviano utilizado para mezcla (con el crudo pesado extraído) así mejorar su calidad para refinación y algunas ocasiones su

*marcelo.salvadorq@epn.edu.ec

Recibido: 08/08/2020

Aceptado: $22 / 09 / 2020$

Publicado: 30/11/2020

$10.33333 /$ rp.vol46n2.01

CC BY 4.0 
transporte. Esta importación en el 2018 ascendió a 32 millones de dólares. Este valor creció en referencia al gasto en 2017 y se prevé un aumento de importaciones de crudo liviano a futuro (EPPetroecuador, 2019a: EPPetroecuador, 2019b). Además, debido a la alta viscosidad que el crudo pesado presenta se generan dificultades operacionales en su transporte, entre ellas se encuentra el aumento en la presión de bombeo de los oleoductos, fallos y taponamiento de la tubería debido a la precipitación o deposición de asfaltenos y reducción en la eficiencia de las bombas (Akbarzadeh et al., 2007, pp. 24-40). Esto conllevará al incremento de gastos de mantenimiento por el fallo continuo o cambio de bombas.

Respecto a la problemática ambiental, se puede señalar a los derrames de petróleo como una de las causas más graves de contaminación. Los elementos naturales como el agua, suelo y aire que interactúan en diferentes ecosistemas son los más afectados debido a la contaminación que se produce por ello. (Plitt, 2010); (Cavazos, Pérez y Mauricio, 2014, pp.112-118). Los derrames de crudo tienen varios orígenes, por ejemplo, el $28 \%$ es provocado por corrosión interna de la tubería, el $26 \%$ por atentados como corte de tuberías para el robo de combustible, el $17 \%$ por fallas mecánicas, el 1,5\% por desastres naturales y el 27,5\% por otras causas (En Ecuador hay un derrame petrolero por semana, 2013; Pacheco, 2019). Algunas de estas causas son consecuencias del transporte de crudo pesado, que genera daños y fallas en los sistemas de transporte y oleoductos (Iturbe, Flores, Castro y Torres, 2007, p.388; Becerra, 2019).

Para la resolución de las dificultades relacionadas con el transporte de crudo pesado existen varias alternativas, de las cuales la mayoría, tiene como objetivo principal la reducción de viscosidad. Algunos de estos métodos se encuentran en proceso de investigación y desarrollo como el flujo núcleoanular o actualización de campo parcial (Abarasi, 2013; Bensakhria, Peysson y Antonini, 2004). Otros, utilizados son el calentamiento de tuberías, la mezcla con naftas o crudos livianos y la adición de aditivos reductores de viscosidad (Peralta, Blanco, Reina y Mantilla, 2017, p.8; Martínez et al, 2011; Langevid, Poteau, Henaut y Argillier, 2004)

El calentamiento consiste en proporcionar calor al oleoducto. La adición de crudo liviano al crudo pesado. Este proceso es generalmente realizado en refinería como una mejora de la calidad del crudo que será utilizado en la obtención de derivados del petróleo como gasolina y diésel. También se usa para facilitar el transporte del mismo (Peralta et al., 2017, pp.812). Estos procesos son usados a nivel mundial. En Ecuador por ejemplo OCP y Petroamazonas los utilizan para el transporte de petróleo.

A pesar de su uso frecuente, estas opciones presentan varias dificultades. El calentamiento, por ejemplo, debe contar con la disponibilidad de energía térmica e incrementa costos de operación, problemas internos de corrosión e inestabilidad en el flujo debido al cambio de las propiedades reológicas (Cortés, 2017, pp.35-52). Por otro lado, la dilución presenta inconvenientes por el aumento de importaciones de crudo liviano, ya que una mezcla efectiva necesitaría una proporción del 20 al $30 \%$ de crudo liviano, con respecto a la cantidad de crudo pesado. Además, el aumento en el caudal por la adición de crudo liviano, daría como resultado un aumento en la presión de bombeo. La mezcla de crudos provocaría inestabilidad en asfaltenos y parafinas lo que provocaría la precipitación de asfaltenos y posteriormente la obstrucción de las tuberías (Zahan, Bjorndalen e Islam, 2004, p.22).

Como último método está el uso de aditivos químicos especializados, diseñados para enfrentar dificultades específicas en los procesos relacionados con el petróleo crudo. Al hablar de aditivos nos referimos a los diferentes tipos de sustancias químicas que se agregan al crudo para mejorar sus propiedades físico químicas. Entre estas sustancias están los reductores de viscosidad, que trabajan para alterar las propiedades tixotrópicas del crudo para crear una viscosidad más baja y facilitan su transporte. Estos químicos pueden aplicarse en cualquier punto del proceso de extracción, transporte y producción de petróleo dependiendo de la necesidad e importancia del mismo. El xileno, hexano, tolueno, algunos tipos de alcoholes, grupos carboxílicos y resinas son algunos aditivos reductores de viscosidad que se conocen (Ancheyta, Trejo y Singh Rana, 2009; Halloran, 2015).

Los asfaltenos constituyen la fracción más pesada del crudo, forman agregados coloidales junto con las resinas. Poseen gran cantidad de anillos aromáticos unidos entre sí. Son una familia de compuestos que varía de petróleo en petróleo pero que poseen un comportamiento común, pueden originar precipitados y determinar las diferentes características del crudo como la viscosidad. Según el contenido de asfaltenos en el crudo, éste será más o menos viscoso (Subiaga y Cuattrocchio, 2006; Delgado, 2006).

Existen varios grupos de aditivos reductores de viscosidad, uno de esos grupos tiene efectos en la fracción de asfaltenos del crudo. Este grupo se clasifica en: inhibidores, disolventes y dispersantes de asfaltenos, cada uno con una acción diferente debido a la variación en sus composiciones químicas. Los inhibidores son moléculas con dos grupos funcionales: uno polar que es el que interacciona con los asfaltenos y otro no polar con un grupo orientado externamente para prevenir la floculación de los asfaltenos, son similares a las resinas naturales y algunos de ellos pueden actuar como dispersantes (Ancheyta et al., 2009).

Los dispersantes son moléculas anfifílicas, aditivos formados principalmente por surfactantes, que estabilizan a las moléculas de asfaltenos. Los disolventes a diferencia de los dos anteriores eliminan depósitos difíciles, modifican la cantidad de anillos aromáticos unidos entre sí y producen un efecto reductivo. Los más comunes son los aditivos formados por compuestos aromáticos como el xileno, el tolueno o los terpenos, los primeros son poco amigables con el ambiente mientras que los terpenos no son tóxicos (Ancheyta et al., 2009; Zamarripa, 2013)

Respecto a las dificultades que los aditivos presentan, está el grado de toxicidad que cada tipo de aditivo pueda tener, existen reductores muy tóxicos tanto para el ambiente como para el ser humano (Zamarripa, 2013). Además, los costos de aditivo varían según el tipo y la cantidad, en algunos casos son costos elevados que no compiten con los otros procedimientos (Allenson, Yen y Lang, 2011; Ghloum et al, 2015). 
En el país, el uso y la investigación de reductores de viscosidad especializados no es tan común, a diferencia de países como Estados Unidos, México, China, Arabia Saudita, Argelia y más; que han aumentado el número de investigaciones con respecto al uso de estos aditivos en la industria petrolera (NCYT Amazings, 2015; Halloran, 2015; Hamed, Kasem y Akbar, 2016; Sinopec Exploration \& Production, 2012, Aisling Chem, 2018).

Algunos de estos químicos ya están en el mercado y son utilizados en los países mencionados anteriormente. Como ejemplo de reductores de viscosidad formulados existe el aditivo inhibidor dispersante de asfaltenos conformado por oxazolidinas derivadas de polialquilos (Mena et al., 2007), al nonilfenol-formaldehído modificado por poliaminas (Hamed, Kasem y Akbar, 2016), dispersantes con base de hexano, los comúnmente utilizados disolventes con base de xileno o escualeno marca Sigma-Aldrich con grandes resultados en los crudos extranjeros (Schlumberger, 2006).

En Ecuador existen empresas que desarrollan aditivos comerciales para el uso en la industria petrolera, algunos probados con éxito y otros aún están en desarrollo (Ekos, 2018). En cuanto a la investigación en el país, es necesario un mayor desarrollo donde se evalué el efecto de este tipo de aditivos reductores de viscosidad disolventes, dispersantes e inhibidores de asfaltenos en el crudo ecuatoriano, tomando como pauta los resultados de los aditivos usados en crudos extranjeros, para el posterior uso y escala a la industria.

El presente trabajo tiene como objetivo el estudio de la reducción de la viscosidad de un crudo pesado para el transporte a través de un oleoducto mediante el uso de compuestos dispersantes y disolventes de asfaltenos, con la finalidad de plantear soluciones a las problemáticas económicas y ambientales anteriormente mencionadas para disminuir las importaciones de aditivos y de crudo liviano utilizados para la reducción de la viscosidad.

\section{METODOLOGÍA}

Sustancias aditivas y reductoras de viscosidad son términos sinónimos. De igual manera, las sustancias reductoras de viscosidad que son inhibidoras, disolventes y dispersantes de asfaltenos son nombradas como términos semejantes con los aditivos o reductores de viscosidad en este documento.

Para el desarrollo de esta investigación se tomaron cinco aditivos comerciales reductores de viscosidad. A partir de ellos, se seleccionó a los que tenían un efecto dispersante, disolvente e inhibidor en la fracción de asfaltenos. Luego se realizaron pruebas para analizar el cambio de propiedades del crudo con los aditivos seleccionados. Se comparó los resultados obtenidos con las características del crudo sin aditivo y se determinó el más eficiente. Finalmente, se realizó la prueba de facilidad de transporte y se determinó al reductor que provee una mejor facilidad de trasporte al crudo por tuberías. Este estudio fue realizado a nivel experimental en laboratorio no en campo.

\subsection{Selección de los reductores de viscosidad}

Los cinco aditivos reductores de viscosidad seleccionados fueron analizados por medio de espectroscopia infrarroja por transformadas de Fourier (FTIR), para su posterior clasificación y selección. Los análisis fueron realizados en el Centro de Investigaciones Aplicadas a Polímeros (CIAP) de la Escuela Politécnica Nacional.

La vibración de los enlaces que conforman los compuestos, son resultado de la absorbancia de energía, dando lugar a un espectro resultante. Cada grupo funcional da lugar a bandas características, que se asignan en la molécula a partes específicas provocando una frecuencia de grupo. Un grupo funcional absorbe radiación sin importar a que está unido, generando una banda y números de onda característica del espectro infrarrojo en un intervalo determinado de frecuencias. De esta manera se puede saber qué grupos funcionales están presentes en los aditivos (Atkins y de Paula, 2009, pp.345-348; Serrano, 2010).

Para el análisis se tomó una muestra del tamaño de una gota de cada reductor. El espectro resultante fue analizado por sus bandas, números de onda y frecuencias características. Este análisis permitió la identificación de los grupos funcionales que forman parte de la composición de cada reductor. (Barraza, de la Rosa, Martínez, Castillo, Cotte y Álvarez, 2013, pp. 370-374).

Con la identificación de los grupos funcionales se clasificó y se seleccionó a los reductores por su efecto disolvente, inhibidor o dispersante de asfaltenos. Los aditivos disolventes de asfaltenos pueden estar formados por grupos funcionales aromáticos, dioles o carboxílicos. Los dispersantes por grupos alquílicos de largas cadenas, sulfonatos o aminas. Los inhibidores por compuestos similares a las resinas naturales (Ancheyta et al., 2009; Zamarripa, 2013)

Los reductores seleccionados se utilizaron para las pruebas de cambio de propiedades físicas y químicas y facilidad de transporte.

\subsection{Análisis del cambio de propiedades físicas y químicas del crudo con los diferentes tipos de reductor estudiados}

Se realizó el análisis de cambio de propiedades físicas y químicas a las muestras de crudo con los tres aditivos seleccionados previamente. Para ello se efectuaron cuatro ensayos a) prueba para determinar la viscosidad b) análisis S.A.R.A., c) prueba de oliensis o mancha, d) prueba para determinar la gravedad API.

Adicionalmente como alternativa a los tres reductores seleccionados, se realizó una mezcla de dos aditivos con diferentes efectos en los asfaltenos, para evaluar la efectividad de los reductores de viscosidad del crudo por medio de la mezcla e individuales. Se tomó como variable la concentración (2 000 y $4000 \mathrm{ppm}$ ) por triplicado, estos valores fueron seleccionados debido a ensayos previos realizados. 
Para el análisis S.A.R.A., la prueba de oliensis o mancha y la prueba para determinar la gravedad API del petróleo se tomaron los mejores resultados en reducción de viscosidad de cada muestra de crudo con aditivo y de la mezcla de dos aditivos.

\subsubsection{Prueba para determinar la viscosidad}

Las pruebas se realizaron en el Laboratorio de Operaciones Unitarias de la Facultad de Ingeniería Química. Su objetivo fue determinar la viscosidad de la muestra de crudo inicial y de las muestras de crudo con los reductores. Se utilizó un viscosímetro rotacional tipo Brookfield, bajo la norma ASTM D2196 "Standard Test Methods for Rheological Properties of Non-Newtonian Materials by Rotational Viscometer" (ASTM, 2018).

Se tomó una muestra de crudo de $250 \mathrm{~mL}$ en un vaso de precipitación que se introdujo en un baño de agua a una temperatura de $45^{\circ} \mathrm{C}$ (temperatura de trabajo normal en un oleducto) (Vivanco, 2011). Se esperó hasta que el crudo llegara a la temperatura de ensayo y ésta fuera constante. A continuación, se ajustó la velocidad de agitación, se desbloqueó la aguja del viscosímetro y se la introdujo dentro de la muestra de crudo. Se esperó hasta que la aguja se estabilizó. Se tomó los datos de viscosidad y porcentaje de torque (ASTM, 2018).

\subsubsection{Análisis estadístico}

Para determinar la relación y eficacia de las pruebas para determinar la viscosidad de las muestras, se realizó el análisis estadístico ANOVA. El análisis tiene como fin determinar si existe o no una relación entre variables (factores) de un estudio, en este estudio las variables seleccionadas fueron la concentración y el aditivo. La relación, se determina por la variabilidad de los resultados entre cada tipo de reductor y las diferentes concentraciones. La viscosidad resultante de las muestras de crudo varía por los factores mencionados, si los valores de viscosidad fueran iguales no existiría una relación y el estudio no tendría valor (Saravia, 2015).

Para el análisis se calculó un valor de significancia (P) entre los factores, éste debió ser menor a 0,05, además se determinó un valor crítico y un valor calculado $\mathrm{F}$ (varianza); si el valor crítico fue menor al valor calculado se confirmaría una relación entre las variables. Estos valores son generales para el análisis y fueron tomados de bibliografía (Saravia, 2015).

Además, para conocer la interacción entre la viscosidad y los reductores se trabajó con un diseño experimental factorial $3 \times 3$ por triplicado, con un grado de confianza del $95 \%$ para observar la relación causa-efecto de los aditivos con la reducción de viscosidad. Fue aplicado a las muestras de crudo sin aditivo y con los 3 aditivos seleccionados previamente (ver la Sección 2.1) donde las variables controladas fueron concentración (1 000, 2000 y 3000 ppm) y aditivo reductor de viscosidad (Abarca, 2016; Cortés, 2017).

\subsubsection{Análisis S.A.R.A. por adsorción cromatográfica}

El análisis S.A.R.A. (Saturados, Aromáticos, Resinas y Asfaltenos), es un procedimiento utilizado para la determinación de las fracciones del crudo. Estás fracciones están conformadas por los siguientes compuestos: resinas, aromáticos, asfaltenos y saturados. Estos ensayos se realizaron bajo la norma ASTM D2007 - 03 "Standard Test Method for Characteristic Groups in Rubber Extender and Processing Oils and Other Petroleum-Derived Oils by the Clay-Gel Absorption Chromatographic Method" (ASTM, 2016a) y se los realizaron en los Laboratorios de Petroamazonas en Shushufindi.

Para el análisis de resultados se tomó los porcentajes de las fracciones de asfaltenos y resinas. Los porcentajes de cada fracción que se obtuvo de la muestra de crudo sin aditivo fueron el blanco. Estas se compararon con las fracciones de las muestras de crudo con los tres diferentes aditivos seleccionados y con la mezcla de dos aditivos.

\subsubsection{Prueba de oliensis o mancha}

Esta prueba fue realizada en el Laboratorio de Operaciones Unitarias de la Facultad de Ingeniería Química de la Escuela Politécnica Nacional. Se determinó la estabilidad de la fracción de asfaltenos que forma parte del crudo. Para la realización de la prueba se utilizó el método de prueba estándar siguiendo la norma ASTM D1370 "Standard Test Method for Contact Compatibility Between Asphaltic Materials (Oliensis Test)" (ASTM, 2016b).

Se tomó $1 \mathrm{~mL}$ de crudo y se mezcló con $1 \mathrm{~mL}$ de xileno. Se colocó una gota de esta mezcla en un papel filtro tipo Whatman de malla \#1. Esta gota fue el blanco y se etiquetó como gota número 1 . Luego se añadió $1 \mathrm{~mL}$ de $\mathrm{n}$-heptano a la mezcla y se colocó una segunda gota en el papel filtro, etiquetada como gota número 2. Se repitió este procedimiento hasta que se completaron 11 gotas. Luego se secó el papel filtro en una estufa a $100{ }^{\circ} \mathrm{C}$ por $15 \min$ (ASTM, 2016b). Después se dejó enfriar el papel y se examinó cada gota para identificar el anillo oleoso formado alrededor de la periferia de cada gota. Se tomó el número de la gota que contiene el anillo oleoso y se comparó con la Tabla 1 (Abarca, 2016).

\subsubsection{Determinación de la gravedad API (Método del hidrómetro)}

Este método de prueba fue realizado en el Laboratorio de Combustibles, Biocombustibles y Aceites Lubricantes (LACBAL) de la Facultad de Ingeniería Química de la Escuela Politécnica Nacional. Este procedimiento se utilizó para determinar la gravedad API y se realizó bajo la norma ASTM D287 "Standard Test Method for API Gravity of Crude Petroleum and Petroleum Products (Hydrometer Method)" (ASTM, 2012).

Se utilizó un hidrómetro dentro del rango API de 11-24 (escala). Se tomó una muestra de $200 \mathrm{~mL}$ de crudo en una probeta de $250 \mathrm{~mL}$ (ASTM, 2012). Luego se introdujo el hidrómetro dentro de la muestra. Se empujó dos divisiones de escala en el líquido y se soltó. Se dejó reposar el hidrómetro dentro de la muestra por un día (ASTM, 2012). Finalmente, se tomó la lectura del valor de gravedad API de la muestra; adicionalmente, se registró el promedio de los valores de temperatura antes y después de la lectura del hidrómetro (ASTM, 2012). 
Tabla 1. Escala para la interpretación del grado de estabilidad de los asfaltenos. (Abarca, 2016)

\begin{tabular}{cc}
\hline Número de gota & Grado de estabilidad \\
\hline $1-3$ & Críticamente inestable \\
$4-6$ & Inestable \\
7 & Umbral \\
$8-10$ & Estables
\end{tabular}

La gravedad API del crudo fue el blanco y se lo comparó con la gravedad API de las muestras de crudo con los tres diferentes aditivos seleccionados y con la mezcla de dos aditivos.

\subsection{Evaluación de la Facilidad del Transporte de Crudo al Trabajar con Reductores de Viscosidad}

Se realizó la evaluación de la facilidad del transporte de crudo en un banco de pruebas de tuberías que puede imitar valores del transporte del crudo por un oleoducto. Las pruebas se realizaron en el Laboratorio de Operaciones Unitarias de la Facultad de Ingeniería Química de la Escuela Politécnica Nacional. En las pruebas se evaluó el efecto de los reductores de viscosidad agregados en cada muestra de crudo, por medio del caudal y presión del sistema.

En la Figura 1 se observa el esquema del banco de pruebas; cuenta con un juego de tuberías Swalegok de $1 / 2$ pulgada de diámetro, una bomba de doble diafragma y desplazamiento positivo de $3 / 8$ de pulgada.

Para el almacenamiento de la muestra se cuenta con un recipiente metálico cilíndrico de 133,40 pulgadas de diámetro, con una capacidad de muestra de $1500 \mathrm{~mL}$, cubierto por una chaqueta de calentamiento acoplada con una termocupla para la medición de la temperatura de la muestra dentro del recipiente.

Las condiciones usadas en el banco de pruebas permiten que los resultados se escalen a nivel industrial. Condiciones como la temperatura que es la misma de trabajo que un oleoducto, la relación entre el caudal y el área transversal de la tubería que es igual a la velocidad del líquido transportado.

Se tomó una muestra de crudo de $1000 \mathrm{~mL}$, en el recipiente para almacenamiento de muestras del equipo. Posteriormente, se encendió el equipo y se trabajó con una temperatura de 45 ${ }^{\circ} \mathrm{C}$ (Vivanco, 2011; Moreira y Mena, 2018). Se abrieron las válvulas que dan paso a la muestra hacia el sistema de tuberías del equipo. Se esperó 2 horas para estabilizar el sistema.

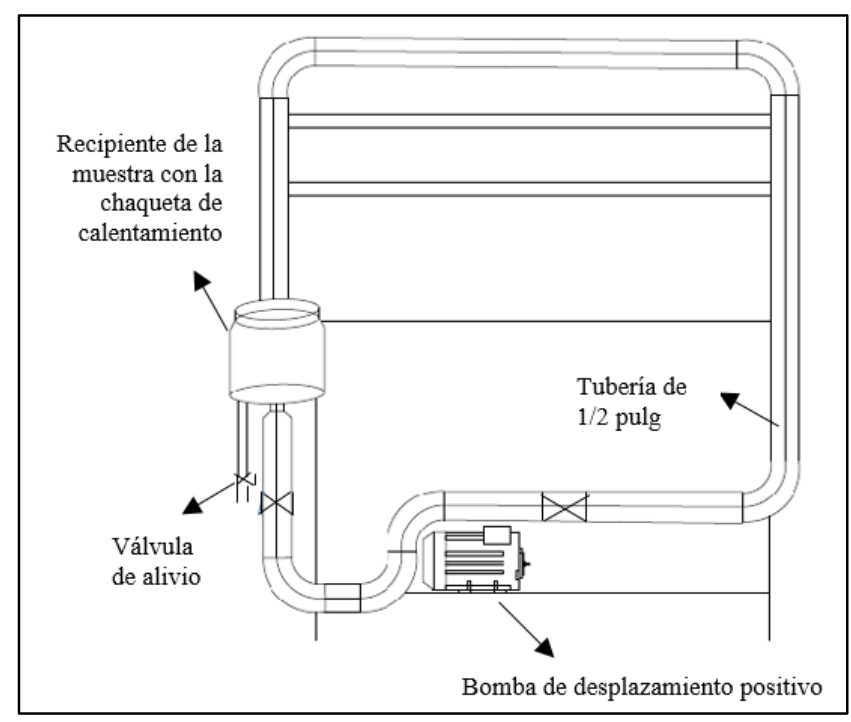

Figura 1. Esquema del banco de pruebas (autoría propia)

Luego se tomaron datos de presión y de caudal de la muestra de crudo (datos que se toman en un oleoducto normal). Para obtener el caudal se tomó datos de volumen del crudo y tiempo de llenado de una probeta graduada (cuatro mediciones). Se repitió este procedimiento para las muestras de crudo con los tres reductores seleccionados y sus mezclas. La lectura de presión se realizó en el manómetro del equipo. Este valor determina la presión que ejerce el fluido dentro de la tubería del equipo para transportarse, al igual que en un oleoducto normal (Cortés, 2017; Abarca, 2016).

Se evaluó la eficacia de los reductores de viscosidad por medio del análisis de la variación de los datos de presión y caudal de la muestra de crudo (blanco) con las muestras modificadas con los distintos tipos de reductores (Cortés, 2017; Abarca, 2016).

\section{RESULTADOS Y DISCUSIÓN}

\subsection{Selección de los reductores de viscosidad utilizados}

El análisis de espectroscopía infrarroja realizado a los reductores dió como resultado los espectros de cada uno con picos y números de onda característicos de los grupos funcionales que los forman. En el estudio de Chavéz, Zamuyo y Barba, (2013) determinaron que el reductor de viscosidad con el que trabajaron era un aditivo dispersante de viscosidad, ya que en su espectro observaron la presencia del grupo succimida con su enlace $\mathrm{C}=\mathrm{O}$ resultado de su banda y número de onda característico. Este grupo se caracteriza por poseer compuestos surfactantes y perteneciente al grupo de dispersores de asfaltenos.

Los reductores seleccionados para las pruebas de cambio de propiedades fisicoquímicas y efectividad de transporte "M01X", "M02-A" y "M03-S". De igual manera que en el estudio anterior, por medio de las bandas y números de onda característicos de los grupos funcionales que conforman cada aditivo se pudo determinar que los aditivos "M01-X" y "M02A" son reductores disolventes de asfaltenos y el aditivo "M03$\mathrm{S}$ " es un reductor dispersante de asfaltenos (Ancheyta, et al., 2009). 
Los reductores de viscosidad "M04-A" y "M05-E" presentaron grupos funcionales como aldehídos, ésteres y sulfocianatos, estos grupos no presentan un efecto dispersante, disolvente o inhibidor de asfaltenos por esta razón no fueron seleccionados, ya que no son motivo de estudio. (Ancheyta et al., 2009; Chavéz et al., 2013).

En la Figura 2., se observa el espectro del aditivo "M01-X", que presenta números de onda (720-680; 810-775; $3005 \mathrm{~cm}^{-1}$ ), siendo estos característicos del grupo funcional aromático; dentro del cual se encuentran compuestos como los isómeros de xileno. Por lo que se clasifica al reductor como un aditivo disolvente de asfaltenos. Al igual que al anterior reductor, el aditivo "M02-A" fue clasificado como disolvente de asfaltenos. En la Figura 3., se observa el espectro del aditivo "M02-A" con números de onda (3 040-2 845; 1 300- 1 250; 1 290-1 265; 1 120-1 $100 \mathrm{~cm}^{-1}$ ) característicos de las bandas de grupos funcionales dioles y carboxílicos (Ancheyta, et al., 2009; QUIORED, 2002).

El reductor "M03-S" fue clasificado como aditivo dispersante de asfaltenos. Como se observa en la Figura 4., este posee números de onda (2 897-2 723; 1 460-1 303; 840-604 cm-1) característicos dentro bandas de grupos funcionales como sulfonatos, aminas y grupos alquílicos de largas cadenas. Además, el espectro analizado tiene similitudes con espectros de crudos de baja viscosidad lo que confirmaría la estabilidad que proporcionaría el aditivo y da como resultado la dispersión de los asfaltenos (Ancheyta et al, 2009; Remolina, Espitia, Luna y Patiño, 2019; QUIORED, 2002).

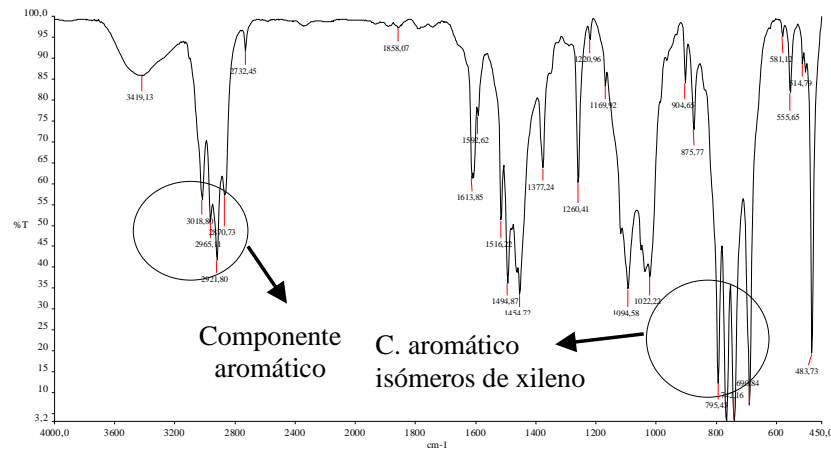

Figura 2. Espectro FT-IR correspondiente al aditivo "M01-X"

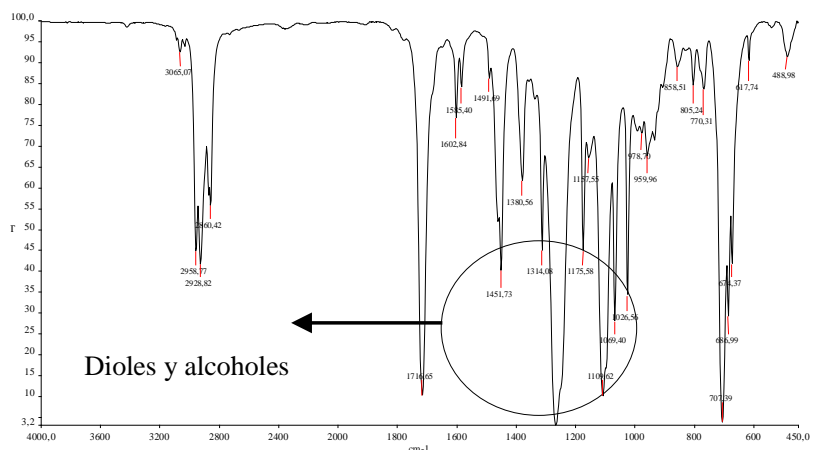

Figura 3. Espectro FT-IR correspondiente al aditivo "M02-A"

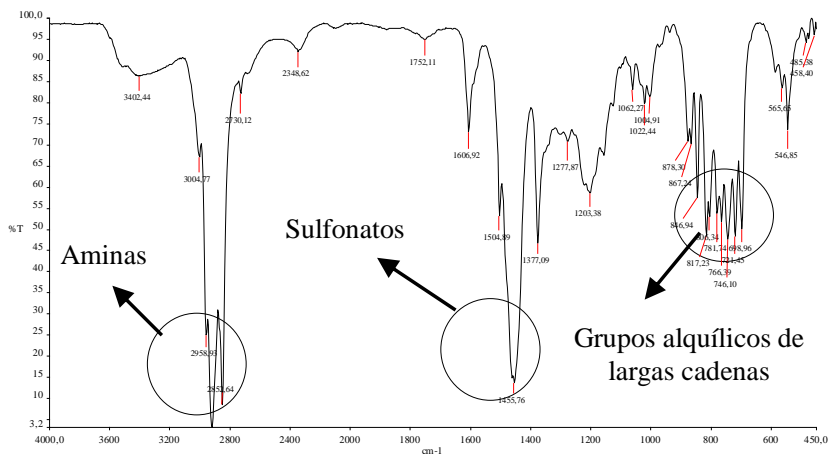

Figura 4. Espectro FT-IR correspondiente al aditivo "M03-S"

\subsection{Análisis del cambio de propiedades físicas y químicas del crudo con los diferentes tipos de reductor estudiados}

\subsubsection{Prueba para determinar la viscosidad}

En la Tabla 2 se presentan los resultados de las pruebas de viscosidad para la muestra de crudo inicial (blanco), las muestras de crudo con los tres reductores seleccionados y con diferentes concentraciones (1 000, 2000 y 3000 ppm). Se observa que el valor de la viscosidad de la muestra de crudo sin aditivo es de $2590,0 \mathrm{cP}$, este valor se tomó como blanco para el análisis. En la Figura 5 se observa que el aditivo "M01-X" posee los porcentajes más altos de reducción de viscosidad en sus tres concentraciones utilizadas con 40,0; 51,0 y 65,0\% para 1000,2000 y 3000 ppm respectivamente, el aditivo "M02-A" posee los porcentajes más bajos de reducción de viscosidad con 26,0; 43,0 y $55,0 \%$ a 1000,2000 y 3000 ppm respectivamente.

Los dos reductores "M01-X" y "M02-A" pertenecen a la misma clasificación de aditivos disolventes de asfaltenos pero su diferente composición y mecanismo de acción hace que la efectividad varíe entre ellos. El aditivo "M01-X" está formado por varios isómeros de xileno. Como se puede comprobar en el espectro. Éstos son los disolventes más efectivos debido a su característica aromática y anfifílica que da como resultado una disolución de asfaltenos más efectiva (Ancheyta et al., 2009) Sin embargo, es tóxico para el ser humano y en el ambiente. Por otro lado, el aditivo "M02-A" está formado por terpenos, principalmente por alcoholes y dioles. Al no poseer anillos aromáticos en su composición, da como resultado un menor porcentaje de reducción de viscosidad en el crudo, pero no son tóxicos (ATSDR, 2016; Remolina et al., 2019, pp. 40, 41).

El mecanismo de acción de los aditivos disolventes de asfaltenos "M01-X" y "M02-A" varía debido a la diferente composición de cada uno. Para el disolvente aromático ("M01X") su acción se da debido a la interrupción de los puentes de hidrógeno y las interacciones intermoleculares $\pi$ en las moléculas de asfaltenos, reduciendo el grado de agregación de las moléculas disolviéndolas. Por otro lado, los disolventes alcohólicos disminuyen el tamaño de los agregados de los asfaltenos por la habilidad de establecer puentes de hidrógeno entre la molécula de disolvente y los asfaltenos disolviéndolos (Remolina et al, 2019, p. 42). 
Tabla 2. Viscosidad de las muestras de crudo sin aditivo y de las muestras de crudo con cada reductor, con diferentes concentraciones y porcentaje de reducción de viscosidad a una temperatura de $45^{\circ} \mathrm{C}$

\begin{tabular}{|c|c|c|c|c|c|c|}
\hline \multicolumn{5}{|c|}{$\begin{array}{l}\text { Viscosidad muestra de crudo (blanco) } \\
\text { (cP) }\end{array}$} & \multicolumn{2}{|l|}{2590,0} \\
\hline & \multicolumn{2}{|c|}{ M03-S } & \multicolumn{2}{|c|}{ M02-A } & \multicolumn{2}{|c|}{ M01-X } \\
\hline $\begin{array}{c}\mathrm{C} \\
(\mathbf{p p m})\end{array}$ & $\mu(\mathbf{c P})$ & $\% \mathrm{Re}$ & $\mu(\mathbf{c P})$ & $\% \mathrm{Re}$ & $\mu(\mathbf{c P})$ & $\% \operatorname{Re}$ \\
\hline 1000 & 1696,0 & 35,0 & 1906,0 & 26,0 & 1557,0 & 40,0 \\
\hline 2000 & 1221,0 & 53,0 & 1482,0 & 43,0 & 1259,0 & 51,0 \\
\hline 3000 & 1025,0 & 60,0 & 1154,0 & 55,0 & 918,0 & 65,0 \\
\hline
\end{tabular}

(\%Re: porcentaje de reducción de viscosidad; $\mu$ : viscosidad; C: Concentración de los reductores)

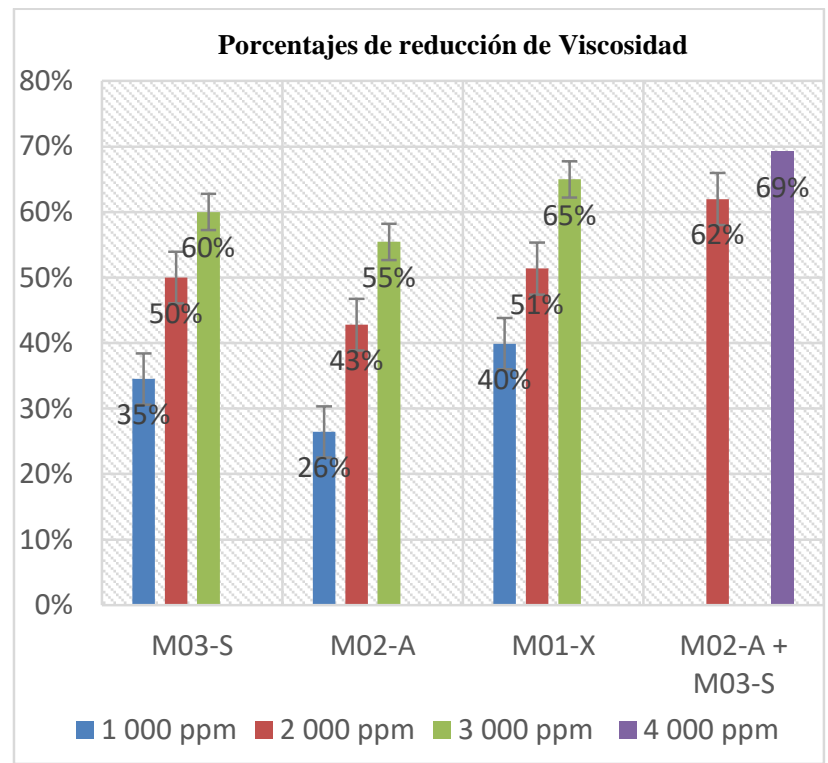

Figura 5. Porcentajes de reducción de viscosidad de las muestras de crudo con cada uno de los aditivos seleccionados M01-X, M02-A y M03-S y la mezcla de dos de ellos (M02-A + M03-S)

Los tres mecanismos de acción tienen como objetivo atacar la agregación de asfaltenos ya sea por medio de la disminución de tamaño y disolución de moléculas o evitando la interacción entre ellas. Por ello la competencia de los mecanismos de acción de los tres aditivos se basa en los compuestos que conforman cada aditivo.

Los más efectivos en reducción de viscosidad son aquellos que poseen anillos aromáticos en su composición como el aditivo "M01-X". El aditivo "M03-S" presenta compuestos alquílicos de cadenas largas y aminas. El primero con una buena efectividad, mientras que el segundo con la efectividad más baja de los grupos de compuesto dando como resultado un porcentaje de reducción intermedio para este aditivo. El aditivo "M02-A" presenta cadenas cortas de compuestos alquílicos (alcohólicos) con una buena efectividad, pero no superior al aditivo "M03-S" (Ancheyta, et al., 2009; Remolina et al, 2019, p. 41).

En comparación con los resultados de reducción de viscosidad en el estudio de Cortés (2017), donde presenta datos de dos aditivos comerciales "solvente orgánico" y "W20" añadidos a muestras de crudo pesado con API similar al utilizado en este estudio.
Los reductores utilizados (M01-X, M02-A y M03-S) obtuvieron mejores resultados en porcentajes de reducción de viscosidad con su concentración más alta. Mientras que los dos aditivos comerciales "solvente orgánico" y "W20" tuvieron rendimientos de $54,8 \%$ y $31,2 \%$ respectivamente; los reductores utilizados en este estudio tuvieron porcentajes de 55,$0 ; 60,0$ y $65,0 \%$.

El aditivo W20 es un compuesto fluidificador y reductor de arrastre que separa el agua emulsionada en la masa del crudo. (OilFlux, 2014). Por otro lado, el "solvente orgánico" es un aditivo de aceite reductor de azufre que ayuda al aumento de la gravedad API. Por todas estas características estos aditivos son usados como reductores de viscosidad y poseen su propio efecto en el crudo. La diferencia de valores presentados anteriormente se da debido a que estos aditivos no tienen como objetivo algún efecto en los asfaltenos. Según bibliografía, los aditivos con un efecto en asfaltenos son los más eficaces con respecto a la reducción de viscosidad en el crudo (Ancheyta et al., 2009; Cortés, 2017).

Según Ancheyta et al., (2009), la combinación de dos tipos diferentes de aditivos da lugar a un efecto reductor de viscosidad más eficiente. En base a esta hipótesis, se realizaron pruebas de determinación de viscosidad de las muestras de crudo agregadas una mezcla, constituida por los reductores "M02-A" y "M03-S" elegidos al ser dos aditivos que presentan un efecto diferente en los asfaltenos y de los tres reductores seleccionados son los menos comunes. En la Tabla 3, se puede observar los resultados de las pruebas de viscosidad realizadas a las muestras de crudo con una mezcla de dos diferentes aditivos (M02-A + M03-S) en dos concentraciones diferentes 2000 y 4000 ppm.

En la Figura 5 se observa el porcentaje de reducción de la mezcla de aditivos (M02-A + M03-S) fue de 61,99 \% Este valor es mayor a los resultantes de los tres aditivos solos; De igual manera, es mayor al porcentaje de reducción de viscosidad de los aditivos comerciales "solvente orgánico" y "W20" analizados en los estudios de Cortés (2017) anteriormente mencionados. La acción reductora de los aditivos se potencia al combinarse, debido a que los reductores atacan de diferente forma a la fracción de asfaltenos del crudo (Remolina et al, 2019, pp. 41, 42).

A partir de estos análisis se tomaron los mejores resultados en reducción de viscosidad de cada aditivo y de la mezcla de aditivos para los siguientes ensayos. Los mejores resultados en reducción de viscosidad se obtuvieron en aditivos con una concentración de 3000 ppm y para la mezcla de dos aditivos (M02-A + M03-S) fue 4000 ppm.

\subsubsection{Análisis estadístico}

Para el análisis estadístico ANOVA se planteó dos hipótesis una nula y otra alternativa. La nula planteó que las medias de los valores de la viscosidad de las muestras de crudo no varían en relación a los diferentes tipos de aditivos y concentraciones. En cambio, la hipótesis alternativa estableció que al menos una media de los valores es diferente. En la Tabla 4, se presentan los resultados del ANOVA, realizado al tipo de aditivo y su concentración. Estos factores se analizaron al tomar en cuenta la viscosidad de las muestras del crudo resultantes. 
Tabla 3. Viscosidad de las muestras de crudo con la mezcla de dos reductores (M02-A + M03-S) y el porcentaje de reducción.

\begin{tabular}{ccc}
\hline Reductor & \multicolumn{2}{c}{ M02-A + M03-S } \\
\hline Concentración $(\mathbf{p p m})$ & $\boldsymbol{\mu}(\mathbf{c P})$ & $\boldsymbol{\%} \mathbf{R e}$ \\
\hline $\mathbf{2 0 0 0}$ & 984,44 & 61,99 \\
$\mathbf{4 0 0 0}$ & 797,22 & 69,22
\end{tabular}

$(\mu$ : Viscosidad)

Tabla 4. ANOVA para la viscosidad-Suma de cuadrados tipo II

\begin{tabular}{cccccccc}
\hline \multicolumn{7}{c}{ Análisis de varianza } \\
\hline Factores & $\begin{array}{c}\text { Suma de } \\
\text { cuadrados }\end{array}$ & $\boldsymbol{G L}$ & $\begin{array}{c}\text { Cuadrado } \\
\text { medio }\end{array}$ & $\begin{array}{c}\text { Razón } \\
\boldsymbol{F}\end{array}$ & $\begin{array}{c}\text { Valor } \\
\boldsymbol{P}\end{array}$ & $\begin{array}{c}\text { Vcr } \\
\boldsymbol{F}\end{array}$ \\
\hline $\begin{array}{c}\text { Concentración } \\
\text { Tipo de } \\
\text { aditivo }\end{array}$ & 714370,13 & 2,00 & 357185,07 & 117,44 & 0,0003 & 6,94 \\
Error & 12161,00 & 2,00 & 58570,50 & 19,26 & 0,0089 & 6,94 \\
Total & 843676,84 & 4,00 & 3041,43 & & & & \\
\hline
\end{tabular}

(GL: grados de libertad; Razón F: varianza generada; Valor P: probabilidad (significación); Vcr F: valor crítico de F)

Los valores de significancia $(\mathrm{P})$ que presentaron las variables de concentración y tipo de aditivo, fueron de 0,0003 y 0,0089 respectivamente, y son menores de 0,05; además, se presentaron valores de F calculada de 177,44 y 19,6 y de F crítica de 6,94 para los dos factores. Estos resultados demuestran que efectivamente existe una relación entre los diferentes tipos de aditivos y concentraciones respecto a los valores de viscosidad de las muestras de crudo comprobando la hipótesis alternativa (Saravia, 2015).

\subsubsection{Análisis S.A.R.A., gravedad API y grado de estabilidad de asfaltenos.}

El análisis de asfaltenos y resinas de las pruebas S.A.R.A., como de gravedad API y de grado de estabilidad de asfaltenos de las muestras de crudo analizadas se realizó en conjunto debido a que estas propiedades tienen relación entre ellas. Además, estos análisis se realizaron como complemento de las pruebas de reducción de viscosidad, para el análisis de cambio de propiedades de crudo. En la Tabla 5 se presentan los resultados de los ensayos.

En algunas investigaciones se puede observar que el uso de este tipo de aditivos reductores de viscosidad de crudo que tienen un efecto inhibidor, disolvente o dispersante de asfaltenos, presentan cambios en las propiedades del crudo. Existe una relación entre la reducción de viscosidad, el aumento de la gravedad API, la disminución de la fracción de asfaltenos y la mejora en su estabilidad. Esto debido a que la disminución de la viscosidad radica en la estabilidad de la fracción de asfaltenos y en la formación de micelas (Rogel, Miao, Vein y Roye, 2015, p.156; Zhang y Huang, 2005, p. 1209).

Cuando las moléculas de asfaltenos se estabilizan la viscosidad del crudo disminuye, debido a que se evita las aglomeraciones de asfaltenos y se da la formación de micelas estabilizantes entre los asfaltenos y las resinas. Por otro lado, la gravedad API aumenta debido a su relación inversa con la densidad. La densidad del crudo disminuye por la separación de sus moléculas y la debilitación de las fuerzas intermoleculares, provocando una expansión en el volumen, siendo este inversamente proporcional a la densidad (Wauquier, 2004).

Tabla 5. Resultados de los ensayos de las muestras de crudo con los 3 aditivos seleccionados y con la mezcla de dos reductores (M02-A + M03-S).

\begin{tabular}{|c|c|c|c|c|c|c|}
\hline \multirow{2}{*}{ Muestra } & \multirow{2}{*}{$\begin{array}{c}\mathrm{C} \\
(\mathbf{p p m})\end{array}$} & \multicolumn{2}{|c|}{ S.A.R.A. } & \multirow{2}{*}{$\begin{array}{c}{ }^{\circ} \mathrm{API} \\
\left(15,5^{\circ} \mathrm{C}\right)\end{array}$} & \multicolumn{2}{|c|}{$\begin{array}{c}\text { Grado de } \\
\text { estabilidad }\end{array}$} \\
\hline & & $\begin{array}{c}\% \\
\text { Resinas }\end{array}$ & $\begin{array}{c}\% \\
\text { Asfaltenos }\end{array}$ & & $\begin{array}{c}\# \\
\text { mancha }\end{array}$ & Grado \\
\hline $\begin{array}{c}\text { Crudo sin } \\
\text { aditivo }\end{array}$ & S/A & 47,59 & 13,11 & 16,0 & 7 & Umbral \\
\hline $\begin{array}{l}\text { Crudo+ } \\
\text { M02-A }\end{array}$ & 3000 & 53,49 & 10,73 & 17,0 & 9 & Estable \\
\hline $\begin{array}{l}\text { Crudo+ } \\
\text { M01-X }\end{array}$ & 3000 & 55,42 & 6,59 & 18,2 & 9 & Estable \\
\hline $\begin{array}{l}\text { Crudo+ } \\
\text { M03-S }\end{array}$ & 3000 & 53,05 & 11,86 & 17,7 & 8 & Estable \\
\hline $\begin{array}{c}\text { Crudo+ } \\
\text { (M02-A + } \\
\text { M03-S) }\end{array}$ & 4000 & 61,88 & 9,27 & 18,5 & 8 & Estable \\
\hline
\end{tabular}

La estabilidad del crudo está dada por la presencia de compuestos aromáticos, cadenas alquílicas laterales cortas, ramificaciones alifáticas, alcoholes, dioles, anillos aromáticos condensados y carbonos cuaternarios que los aditivos reductores de viscosidad le aportan. Por ejemplo, al incrementar la cantidad de compuestos con anillos aromáticos, aumenta la sustitución en cadenas ramificadas de alifáticos y genera una mayor estabilidad (Yu et al., 2014, p. 379).

Como se observa en la Tabla 5 y en la Tabla 2, la relación entre la reducción de viscosidad, el aumento de la gravedad API, la disminución de la fracción de asfaltenos y la mejora en su estabilidad es visible en los valores de las pruebas realizadas a las muestras. Por ejemplo, cuando a la muestra de crudo se le agregó el aditivo "M01-X" sus propiedades variaron, como se observó en la Sección 3.2.1, fue el aditivo más efectivo, con una mayor reducción de viscosidad en el crudo, la gravedad API aumentó de 16,0 a 18,2; el porcentaje de la fracción de asfaltenos se redujo de $13,11 \%$ a $6,59 \%$ y su estabilidad paso del umbral a estable.

Los resultados de las pruebas realizadas al "solvente orgánico" en los estudios de Cortés (2017, pp. 78 y 79) se observa que entre más alto era el porcentaje de reducción de viscosidad, mayor el aumento de la gravedad API. Se presentó una reducción de viscosidad de 54,84 \% con un aumento de API de 14,7 a 15,7. Resultados que al compararlos con los obtenidos con los tres aditivos utilizados (M01-X, M02-A y M03-S) son menores a los de este estudio.

Hay que tomar en cuenta que el crudo es un fluido no newtoniano. Este presenta flujos turbulentos, el aditivo en el crudo hace que tienda a comportarse más como un fluido newtoniano. Se debe tomar en cuenta a la velocidad de corte del fluido ya que permite la relación de estas características con la viscosidad (García et al., 2010; Serway, Foughn, 2001).

El porcentaje de fracción de resinas en la muestra también es un factor determinante al evaluar la reducción de viscosidad asociada a la cantidad de asfaltenos. Las resinas alteran la capacidad de agregación e interacción de los asfaltenos, lo que 
provoca su inhibición. Al aumentar la cantidad de resinas, mayor será la reducción de viscosidad (Zamarripa, 2013).

La muestra de crudo con una mayor cantidad de resinas fue la que contenía el aditivo "M02-A". Al no ser un fuerte disolvente de asfaltenos, como el reductor "M01-X", complementa su efecto reductor de viscosidad con un aumento en el porcentaje de la fracción de resinas en la composición del crudo. Como resultado, se tiene que la diferencia del porcentaje de reducción de viscosidad entre los aditivos "M01$\mathrm{X}$ " y "M02-A" es alrededor de 10 y demuestra que los dos aditivos son reductores de viscosidad igualmente eficientes.

Respecto a los resultados de las muestras de crudo con la mezcla de dos aditivos (M02-A + M03-S) al igual que en las pruebas de viscosidad, la mezcla presenta los mejores resultados en comparación con los aditivos analizados. El porcentaje de la fracción de asfaltenos fue de 9,27\% y de resinas de $61,88 \%$ debido a que la mezcla posee una acción combinada de disolución y dispersión de asfaltenos que se complementan con el aumento del porcentaje de la fracción de resinas. Esto resulta en un incremento de gravedad API de 16,0 a 18,2 y con un grado estable de asfaltenos.

\subsection{Evaluación Técnica de la Facilidad del Transporte de Crudo al Trabajar con Reductores de Viscosidad}

En el transporte de crudo por un oleoducto se presentan varios parámetros para evaluar la efectividad de transporte, entre ellos la presión del sistema, la temperatura, el caudal del crudo, etc. Este último es uno de los más importantes (Oñate y Rodríguez, 2012). Por ello se analizó el caudal de las muestras de crudo sin aditivo, con los tres diferentes reductores de viscosidad (M01-X, M02-A y M03-S) y con su mezcla (M02$\mathrm{A}+\mathrm{M} 03-\mathrm{S})$ en un banco de pruebas que presenta comportamientos similares al transporte de crudo por un oleoducto.

En la Tabla 6 se observan los resultados obtenidos en el banco de pruebas del caudal de las muestras de crudo con y sin aditivo y de la presión del sistema. Las muestras de crudo con caudales más altos y con presiones del sistema más bajos son las muestras a las que se les agregó el aditivo "M01-X" y la mezcla de los aditivos (M02-A+M03-S), con un caudal de crudo sin aditivo de $1,24 \mathrm{~mL} / \mathrm{s}$ y presión de $60 \mathrm{psi}$, a un caudal de 2,03 y $2,72 \mathrm{~mL} / \mathrm{s}$ y presión del sistema de 42 y 40 psi respetivamente.

En comparación con la investigación de Zambrano (2015), donde se observa un perfil de crudos pesados con diferentes gravedades API y sus caudales en un oleoducto. Un crudo con un API de 13,5 tiene un caudal de 5680 bopd y un crudo de 14,5 API un caudal de 25363 bopd. Al igual que los valores resultantes de este estudio presentan una disminución de la gravedad API y un aumento del caudal.

El aumento de caudal depende de la facilidad que tiene el crudo para transportarse. Facilidad que está dada tanto por la viscosidad como por la gravedad API. Estos valores pueden cambiar dependiendo de la química del crudo (Oñate y Rodríguez, 2012).
Tabla 6. Caudal y presión del sistema para las muestras de crudo sin aditivo, con los 3 aditivos seleccionados y con la mezcla de dos reductores (M02-A +M03-S) y, para la evaluación de la facilidad de transporte.

\begin{tabular}{cccc}
\hline Muestra & $\begin{array}{c}\text { Concentración } \\
(\mathbf{p p m})\end{array}$ & $\begin{array}{c}\text { Caudal } \\
\text { promedio } \\
(\mathbf{m L / s})\end{array}$ & $\begin{array}{c}\text { Presión del } \\
\text { sistema (psi) }\end{array}$ \\
\hline Crudo sin aditivo & S/A & 1,24 & 60 \\
Crudo+ M02-A & 3000 & 1,60 & 50 \\
Crudo+ M01-X & 3000 & 2,03 & 42 \\
Crudo+ M03-S & 3000 & 1,80 & 46 \\
$\begin{array}{c}\text { Crudo+ (M02-A + } \\
\text { M03-S) }\end{array}$ & 4000 & 2,72 & 40 \\
\hline
\end{tabular}

Estos resultados se dan por medio de diferentes factores, el efecto que provocan los aditivos al reducir la viscosidad en el crudo es asegurar un flujo de transición y retrasar la aparición de remolinos turbulentos (Johnston, Lauzon y Pierce, 2008, pp. 89-92).

El crudo, al ser un fluido no newtoniano, no se rige por la ley de Poiseuille, la cual relaciona indirectamente el caudal con la viscosidad del fluido, debido a que se acopla a fluidos ideales con flujo laminar, pero esta relación permite entender el efecto que provoca el aditivo en el crudo. Al agregar un reductor de viscosidad al crudo se logra estabilizarlo y que no presente un flujo turbulento al transportarse por la tubería; es decir, se asemeja a un flujo laminar donde existe una menor fricción con las paredes del oleoducto. También el aditivo hace que el petróleo tienda a comportarse más como un fluido newtoniano, lo que genera una mayor facilidad de transporte y da como resultado final un aumento en el caudal por la reducción de viscosidad (García et al., 2010; Serway, Foughn, 2001; Alfonso, Hernández, Mediaceja, 2017).

La presión del sistema en el banco de pruebas es la presión que ejerce la bomba para transportar la muestra por la tubería. Se relaciona con la viscosidad debido a que ésta es la resistencia a fluir, y entre más alta sea la viscosidad de la muestra, mayor es la resistencia a fluir y, por ende, la presión necesaria para bombear el flujo es más alta debido al impedimento de estas fuerzas para fluir. Además, los fluidos viscosos presentan pérdidas de energía en forma de calor debido a la fuerza que ejerce el fluido al pasar por las paredes de la tubería y, por tanto, la velocidad de salida de la tubería y caudal son más bajos (Streeter, 2003; Rosero, 2012; Martín, Salcedo y Font, 2011).

Los resultados obtenidos en este estudio pueden escalarse a nivel industrial ya que la relación entre el caudal del crudo y el área de la tubería tiende a ser la misma y la presión de bombeo es dada por la facilidad de transporte (viscosidad). Además, este estudio puede servir como base para la formulación de aditivos reductores de viscosidad. 


\section{CONCLUSIONES}

Por medio de la selección física y química de los reductores de viscosidad se determinó los tres aditivos seleccionados para ser utilizados: "M01-X", "M02-A" y "M03-S". Se los clasificó como aditivos reductores de viscosidad los dos primeros y como disolvente de asfaltenos el último, debido a la composición química de cada uno identificada en los ensayos FT-IR.

De los tres aditivos, el más eficiente en cuanto a reducción de viscosidad del crudo fue el aditivo "M01-X" con un porcentaje de reducción de 65,0 \% a una concentración del aditivo de $3000 \mathrm{ppm}$. Respecto a las muestras de crudo agregadas la mezcla de los aditivos (M02-A+M03-S), se tuvo como resultado una reducción de viscosidad mayor a las muestras que contenían solo un aditivo, con 69,2 \% de reducción.

La adición de los tres reductores de viscosidad y la mezcla de los reductores (M02-A+M03-S) mejoraron la calidad del crudo, aumentó la gravedad API y contribuyó a la estabilidad de la fracción de asfaltenos. La mezcla de aditivos fue la que tuvo un cambio de propiedades más favorable, con un aumento de gravedad API de 16,0 a 18,5; con una reducción del porcentaje de la fracción de asfaltenos de $13,11 \%$ a 9,27 \% y con mejora de la estabilidad de los asfaltenos y, por tanto, del crudo.

Con respecto a la evaluación de la facilidad de transporte, el aditivo "M01-X" y la mezcla de los aditivos (M02-A y M03-S) tuvieron los mejores resultados, con un aumento del caudal de las muestras de crudo en el banco de pruebas, desde un caudal de crudo sin aditivo de $1,242 \mathrm{~mL} / \mathrm{s}$ a un caudal de 2,030 y $2,717 \mathrm{~mL} / \mathrm{s}$ respetivamente. Además, se tuvo como resultado la disminución de la presión del sistema de 60 psi a 42 y 40 psi respectivamente.

\section{REFERENCIAS}

Abarasi, H. (2013). A review of technologies for transporting heavy crude oil and bitumen via pipelines. Journal of Petroleum Exploration and Production Technology. 4(3). DOI: 10.1007/s13202-013-0086-6

Aisling Chem. (2018). Reductores de viscosidad para petróleo pesada. Recuperado de: https://www.aislingchem.com/es/products/reductores -de-viscosidad-para-petroleo-pesado/ . Junio, 2019

Abarca, A. (2016). Estudios del efecto de reductores de viscosidad en crudo pesado (tesis de pregrado). Escuela Politécnica Nacional, Quito, Ecuador.

Akbarzadeh, K., Hammami, A., Kharrat, A. Z., Allewnson, S., Creek, J., y Solbakken, T. (2007). Los asfaltenos: Problemáticos pero ricos en potencial. OilField Review, 24-47. Recuperado de: https://www.slb.com/-/media/files/oilfieldreview/p22-43-spanish . Julio, 2019

Alfonso, H., Hernández, A., y Mediaceja, Y. (2017). Modelado de la viscosidad aparente de un petróleo crudo de $11^{\circ} \mathrm{API}$ con comportamiento no newtoniano.
Ingeniare. 25 (4). http://dx.doi.org/10.4067/S071833052017000400674

Allenson, S., Yen, A., y Lang F. (2011). Application of Emulsion Viscosity Reducers to Lower Produced Fluid Viscosity. Proceedings of the Annual Offshore Technology Conference 1. DOI: 10.4043/22443-MS

Ancheyta, J., Trejo, F., y Singh Rana, M. (2009). Asphaltenes: chemical transformation during hydroprocessing of heavy oils (1era. ed). Puerto España, Trinidad y Tobago: Taylor \& Francis Group

Ardilla, J., y Pacheco, J. (2017). Análisis del comportamiento de la viscosidad de un crudo extrapesado con la aplicación de reductores de viscosidad, polímeros, derivados del petroleo y productos orgánicos. Universidad Industrial de Santander. Bucaramanga, Venezuela

ASTM. (2012). ASTM D287 “Método de prueba estándar para determinar la gravedad API del petróleo crudo y productos derivados del petróleo (Método del Hidrómetro)". Recuperado de: https://compass.astm.org.bvirtual.epn.edu.ec/ download/D287-SP.1403.pdf. Agosto, 2019

ASTM. (2016a). ASTM D 2007 - 03 “'Standard Test Method for Characteristic Groups in Rubber Extender and Processing Oils and Other Petroleum-Derived Oils by the Clay-Gel Absorption Chromatographic Method". Recuperado https://compass.astm.org/download/D200703.6762.pdf. Agosto, 2019

ASTM. (2016b). ASTM D 1370 /D1370M “Standard Test Method for Contact Compatibility Between Asphaltic Materials (Oliensis Test)". Recuperado de: https://compass.astm.org.bvirtual.epn.edu.ec/downlo ad/D1370D1370M.31845.pdf. Agosto, 2019

ASTM. (2018). ASTM D2196 "Standard Test Methods for Rheological Properties of Non-Newtonian Materials by Rotational Viscometer". Recuperado de: https://compass.astm.org/EDIT/html_annot.cgi?D21 96+18e1. Agosto, 2019

Atkins, P., y de Paula, J. (2009). Elements of Physical Chemistry. 5ta edición. W.h. Freeman.

ATSDR (2016). ToxFAQs-Xileno(Xylene). Recuperado de https://www.atsdr.cdc.gov/es/toxfaqs/es_tfacts71.ht ml. Agosto, 2019

Banco Central del Ecuador. (2018). Reporte del sector petrolero II trimestre de 2018. Recuperado de: https://contenido.bce.fin.ec/documentos/Estadisticas/ Hidrocarburos/ ASP201806.pdf Quito. Julio, 2019

Barraza, G., de la Rosa, L., Martínez, A., Castillo, H., Cotte, M., y Alvarez, E. (2013). La microespectroscopía de infrarrojo con transformada de Fourier (FTIRM) en el estudio de sistemas biológicos. Revista Latinoamericana de Química. 41 (3). Recuperado de: http://www.scielo.org.mx/scielo.php?script=sci_artte xt\&pid=S0370-59432013000300001. Agosto, 2019 
Becerra, S. (2019). Nuestro vivir en la Amazonía ecuatoriana: entre la finca y el petróleo. Quito: ABYA AYALA.

Bensakhria, A, Peysson, Y., y Antonini, G. (2004). Experimental study of the pipeline lubrication for heavy oil transport. Oil \& Gas Science and Technology. 59(5), 523-533 .DOI: 10.2516/ogst:2004037.

Briceño, M. (2007). Evaluación de los reductores de viscosidad en crudos pesados de occidente (tesis de pregrado). Universidad Rafael Urdaneta, Maracaibo, Venezuela.

Cavazos, J., Pérez, B., y Mauricio, A. (2014). Afectaciones y consecuencias de los derrames de hidrocarburos en suelos agrícolas de Acatzingo, Puebla, México. Agricultura, sociedad y desarrollo. 11 (4). Recuperado de: https://www.researchgate.net/publication/317441991 _Afectaciones_y_consecuencias_de_los_derrames_d e_hidrocarburos_en_suelos_agricolas_de_Acatzingo Puebla_Mexico/fulltext/59eea833aca272029ddf7a0 5/Afectaciones-y-consecuencias-de-los-derrames-dehidrocarburos-en-suelos-agricolas-de-AcatzingoPuebla-Mexico.pdf. Agosto, 2019

Chavéz, T., Zamudio, L., y Barba, V. (2013). Aromatic polyisobutylene succinimides as viscosity reducers with asphaltene dispersion capability for heavy and extra-heavy crude oils. Enegy and fuels. 27(4), 19942001. DOI: https://doi.org/10.1021/ef301748n

Cortés, C. (2017). Técnicas para mejorar el transporte de crudos pesados por oleoductos (tesis de pregrado). Escuela Politécnica Nacional, Quito, Ecuador.

Delgado, J. (2006). Asfaltenos: composición, agregación, precipitación.

Recuperado de http://www.firp.ula.ve/archivos/cuadernos/S369A.pd f. Julio, 2019

Ekos. (2018). Guia de negocios: Ekos. Obtenido de Ekos Web site:

https://www.ekosnegocios.com/novip/championtechnologies-del-ecuador-sa-championtech. Julio, 2019

En Ecuador hay un derrame petrolero por semana. Diario El Universo. (2019) Recuperado de https://www.eluniverso.com/noticias/2014/06/11/not a/1026781 /ecuador-hay-derrame-petrolero-semana. Agosto 2019

EPPetroecuador. (2019a). Informe estadístico EneroDiciembre 2018. Ecuador: Jefatura Corporativa de Planificación.

EPPetroecuador. (2019b). Informe estadístico Enero-Marzo 2019. Ecuador: Jefatura Corporativa de Planificación.

García, C., Cáceres, O., Hernández, F., Afanador, L., Rodríguez, L., Casallas, P., y Cruz, G. (2010). Optimización del Transporte por Oleoducto de Crudo Pesado Castilla. El Reventón Energético. 8(1). 21-27. Recuperado http://revistas.uis.edu.co/index.php/revistafuentes/art icle/viewFile/1148/1540. Marzo, 2020

Ghloum, E., Rashed, A., Al-Jasmi, A., Al-Ali, A., Mali, P., y Sanyal, A. (2015). Selection of Suitable Viscosity Reducer to Facilitate Test Production of Heavy Oil and Deep Reservoir. Society of Petroleum Engineers. DOI: https://doi.org/10.2118/175312-MS.

Gurlf Professional. (2002). Pipeline Rules of Thumb Handbook: Quick and accurate solutions to your everyday pipeline problems. E.W.McAllister.

Halloran, M. (2015). Incorporative Production Additives Lower HSE Concerns \& Improve Processes. UPSTREAM PUMPING. Recuperado de: http://www.upstreampumping.com/article/2015/incor porative-production-additives-lower-hse-concernsimprove-processes/ Mayo, 2020.

Hamed, F., Kasem, H., y Akbar, V. (2016). A comprehensive experimental evaluation of asphaltene dispersants for injection under reservoir conditions. Petroleum Science, 280-291. DOI 10.1007/s12182-016-0078-5

Iturbe, R., Flores, C., Castro, A., y Torres, L. (2007). Sub-soil contamination due to oil spills in zones rrounding oil pipelines-pump stations and oil supipelines right-ofways in Southwest-Mexico. Environ Monit Assess, 387-398. DOI: https://doi.org/10.1007/s10661-0069593-y.

Johnston, R., Lauzon, P., y Pierce, J. (2008). Enhance the flow and reduce the drag. Hydrocarbon Engineering. 8992. Recuperado de: https://www.researchgate.net/publication/293706928 _Enhance_the_flow_and_reduce_the_drag. Marzo, 2020.

Langevin, D., Poteau, S., Henaut, I., y Argillier, J. (2004). Crude oil emulsion properties and their application to heavy oil transportation. Oil \& Gas Science and Technology. 59(59), 511-521. DOI: 10.2516/ogst:2004036.

Martín, I., Salcedo, R., y Font, R. (2011). MECÁNICA DE FLUIDOS:Tema1. Flujo interno de fluidos incompresibles y compresibles. Recuperado de: https://rua.ua.es/dspace/bitstream/10045/20299/1/te ma1_Flujo\%20interno.pdf. Marzo, 2020.

Martínez, R., Mosqueira, M., Zapata, B., Mar, E., Bernal, C., Clavel, J., Aburto, J. (2010). Transportation of heavy and extra-heavy crude oil by pipeline: A review. Journal of Petroleum Science and Engineering. 75(34) DOI: https://doi.org/10.1016/j.petrol.2010.11.020

Mena, V., López, S., Zamudio, L., Douda, Y., Lozada, M., Morales, A.,. . Bárcenas, M. (2007). México Patente $\mathrm{n}^{\circ}$ WO2009078694A1.

Moreira, A. y Mena, S. (2018). Análisis de las Condiciones de Operación en Estado Estable de Estaciones Reductoras de Presión en Oleoductos. Revista Politécnica. 42(1). 47-52. Recuperado a partir de 
https://revistapolitecnica.epn.edu.ec/ojs2/index.php/r evista_politecnica2/article/view/970

NCYT Amazings. (2015). Quimica: Científicos mexicanos logran reducir la viscosidad de crudos pesados en un 90\%. Obtenido de NCYT Amazings web site: https://noticiasdelaciencia.com/art/13352/cientificosmexicanos-logran-reducir-la-viscosidad-de-crudospesados-en-un-90-. Julio 2019

Oñate, J., y Rodriguez, R. (2012). Evaluación de las alternativas de transporte de crudo pesado por tuberías: caso aplicado al campo Rubiales. Universidad Industrial de Santander. Bucaramanga, Venezuela

OilFlux. (2014). Resultados de la Prueba W20. Recuperado de:http://www.oilfluxamericas.com/extraction-wellstestresults-w20-sp.php. Mayo, 2020.

Pacheco, M. (5 de junio de 2019). Contaminación por derrame de crudo del bloque Yuralpa, en Napo. Diario El Comercio. Obtenido de https://www.elcomercio.com/actualidad/contaminaci on-derrame-crudo-napo-petroamazonas.html. Agosto, 2019.

Peralta, A., Blanco, J., Reina, J., y Mantila, L. (2017). Transporte de crudo pesado por oleoducto usando el método de dilución: Un enfoque práctico para modelar la caída de presión y la precipitación de asfaltenos. El reventón energético. 15(2), 7-17. DOI: https://doi.org/10.18273/revfue.v15n2-2017001 (Agosto, 2019)

Plitt, L. (2010). ¿Cuál es el verdadero impacto de un derrame de petróleo?. Noticiero de la BBC Mundo, Medio Ambiente. Obtenido de: https://www.bbc.com/mundo/internacional/2010/04/ 100428_derrame_petroleo_claves_lp. Agosto, 2019.

Producción del ITT es $80 \%$ agua y 20\% petróleo. (2018). Diario El Universo. Recuperado de https://www.eluniverso.com/ noticias/2018/11/19/nota/7057962/produccion-itt-es80-agua-20-petroleo. Julio, 2019

QUIRED. (2019). Absorciones IR para grupos funcionales representativos. Recursos educátivos de química orgánica. Recuperado de https://www.ugr.es/ q quiored/lab/tablas_espec/ir.htm. Marzo, 2020.

Remolina, S., Espitia, J., Luna, C., y Patiño, E. (2019). Una mirada al desarrollo de aditivos reductores de viscosidad y sus aplicaciones en el transporte de crudos pesados. Revista Ion. 32(1). 35-48. DOI: http://dx.doi.org/10.18273/revion.v32n1-2019003

Rogel, E., Miao, T., Vien, J., y Roye, M. (2015). Comparating asphaltenes deposit versus crude oil. Fuel. 147, 155160. DOI: https://doi.org/10.1016/j.fuel.2015.01.045

Rosero, P. (2012). Desarrollo de un algoritmo y programa en MATLAB para sistematizar y automatizar un modelo digital hidrodinámio en estado estable de un oleoducto para transporte de crudo liviano (tesis de postgrado). Escuela Politécnica Nacional, Quito, Ecuador.

Saravia, J. (2015). Dos caminos, un destino: el ANOVA de dos vías. Recuperado de Stats SOS web site: https://statssos.net/2015/03/30/dos-caminos-undestino-el-anova-de-dos-vias/. Agosto, 2019

Schlumberger. (2006). La importancia del petróleo pesado. Recuperado de: https://www.slb.com//media/files/oilfield-review/heavy-oil-3-spanish. Mayo, 2020

Secretaría de Hidrocarburos . (2017). Cifras Estimadas de Reservas de Petróleo por Categoría. Ecuador .

Serway, R., y Faughn, J. (2001).Física. (5ta Edición). Pearson Educación de México, S.A. Ciudad de México, México.

Serrano, J. (2010).Curso: Instrumentación y métodos de análisis químico. Posgrado en Ingeniería del agua y del terreno. Recuperado de https://www.upct.es/ minaeees/espectroscopia_infra rroja.pdf Mayo, 2020.

Sinopec Exploration \& Production. (2012). Reductor de viscosidad bifásico para crudos pesados. Recuperado de:

http://www.iapg.org.ar/seccionalsur/NUEVA/Jornad as2012/Trabajos/21-

Reductor\%20de\%20Viscosidad\%20Bifasico\%20par a\%20crudos\%20pesados.pdf. Junio, 2019

Subiaga, A., y Cuattrocchio, A. (2006). Partes fundamentales y reología de asfaltos para uso vial. Centro de Investigaciones Viales UNT Reg. La Plata. Argentina. Recuperado de: https://web.archive.org/web/20061208170635/http:// www.frlp.utn.edu.ar/lemac/Publicaciones/Del\%2020 05/007\%20-\%20cila\%20reologia.pdf. (Agosto,2019)

Streter, V. (2003). Mecánica de Fluidos. (9na Edición). Ediciones Castillo SA, Madrid, España.

Vivanco, A. (2011). Modelación del tramo Páramo-Puerto Quito del OCP S.A (Oleoductos de Crudos Pesados), mediante el programa Pipelinestudio (tesis de postgrado). Escuela Politecnica Nacional, Quito, Ecuador.

Wauquier, J. (2004). El Refino ddel Petróleo: Petróleo Crudo, Productos Petrolíferos, Esquemas de Fabricación. Ediciones Díaz de Santos, Madrid, España.

Yu, G., Karinshak, K., Harwell, J., Grady, B., y Ghosh, M. (2014). Interfacial behevior and water solubility of various asphaltenes at high temperature. Colloids and surfaces A: Physicochemical and Engineering Aspects. 441. 378-388. DOI: https://doi.org/10.1016/j.colsurfa.2013.09.020

Zahan, M., Bjorndalen, N., e Islam, M. (2004). Detection of precipitation in pipelines. Petrol. Sci, 22. DOI: https://doi.org/10.1081/LFT-200034063 
Zamarripa, O. (2013). Determinación de una correlación para obtener la distribución de pesos moleculares de agregados de asfaltenos a partir de la caracterización de aceites crudos y sus mezclas (tesis de pregrado). Instituto Politécnico Nacional, México D.F., México. págs., 28-30

Zambrano, M. (2015). Aseguramiento de flujo en el transporte de petróleo pesado - disminución de caudal. Universidad Central del Ecuador, Quito, Ecuador.

Zhang, S., y Huang, H. (2005). Geochemistry of Palaeozoic marine petroleum from the Trin Basin, NW China: Part 1. Iol family classification. Journal of the European Association of Organic Geochemistry. 36. 1204-1214 DOI:10.1016/j.orggeochem.2005.01.013

\section{BIOGRAFÍAS}

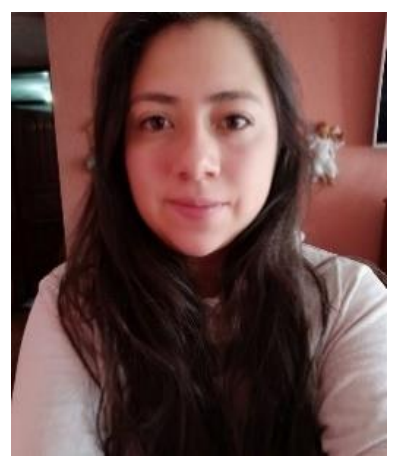

Viviana Isabel Piedra Montoya. Ingeniera química graduada de la Escuela Politécnica Nacional 2020. Área de investigación en reductores de viscosidad para crudo pesado y con experiencia en el campo ambiental. Trabajé como asistente técnica en la Fundación Futuro Latinoamericano para el Proyecto Plan de Acción para el componente de Adaptación de la NDC. ORCID ID: https://orcid.org/0000-0002-7380-1648

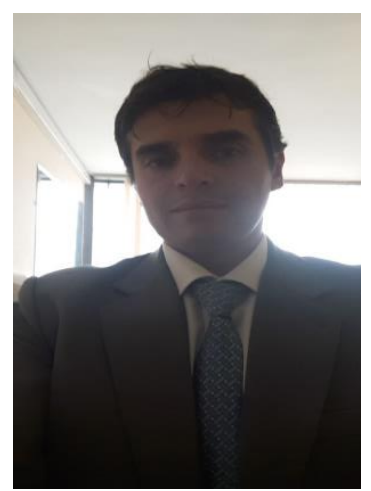

Marcelo Fernando Salvador Quiñones. Ingeniero químico graduado de la EPN 2006 y Máster en sistema de transporte de petróleo y sus derivados graduado de la EPN 2012. Trabajé como analista de metalografía y corrosión en el Laboratorio de Metalografía de la EPN desde 2009 hasta 2012. Trabajé en el sector petrolero como inspector QAQC de tuberías de pozo y perforación, herramientas de perforación, cementación y completación para Petroamazonas. Desde octubre del 2014 trabajo en la EPN como docente y jefe del Laboratorio de Operaciones Unitarias de la Facultad de Ingeniería Química. Identificador ORCID: 0000-0003-46133311

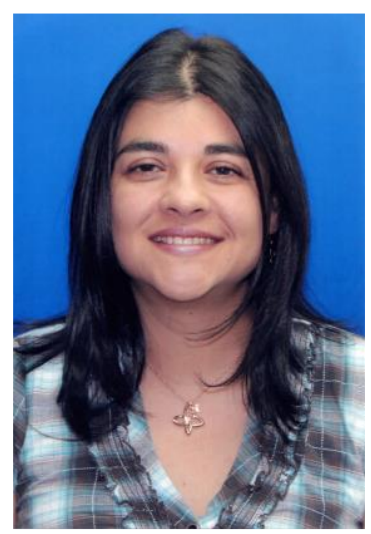

Liliana Guzmán Beckmann. Ingeniera Química de la Escuela Politécnica Nacional (EPN), Quito, Ecuador. Master en Diseño de Procesos, Universidad Central del Ecuador (UCE). Ingeniera de campo de registros eléctricos en pozos en perforación y en producción de petróleo, Baker Hughes. Gerente de Logística y Procesos para trazar el combustible ecuatoriano para prevenir y controlar el contrabando y desvío de derivados del petróleo, Decipher C.A. Actualmente, profesora del Departamento de Ingeniería Química de la EPN, investiga temas relacionados con petróleo, combustibles y biocombustibles. ORCID: 0000-0003-1623-4015

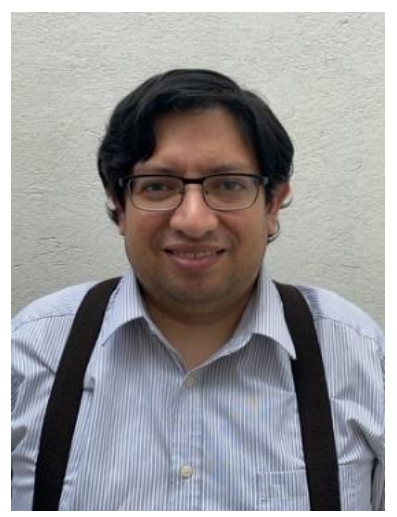

Roque Santos. Nació en Quito en 1983. Se graduó como ingeniero químico de la Escuela Politécnica Nacional en marzo de 2009. En el 2010 obtuvo la maestría en Ingeniería Nuclear y Aplicaciones por la Universidad Autónoma de Madrid. Se incorporó en el año 2010 a la Escuela Politécnica Nacional como docente del Departamento de Ciencias Nucleares. En el año 2017 obtiene su Doctorado en Ingeniería Nuclear en la Universidad de Tennessee en Knoxville. La investigación del Dr. Santos se centra en la detección de radiaciones aplicada a la Ingeniería Química. Número ORCID: 0000-0002-4202-5818



José Iván Chango Villacís. Especialista en Técnicas de Análisis Químicos CIAP-EPN. Doctor en Química, Magister en Química Analítica por la Universidad Central del Ecuador, Asesor de empresas privadas en el ámbito de formulaciones de cauchos. Número Orcid: 00000002-4391-5775 
